Service social

\title{
Engagement social, engagement identitaire - Parcours de femmes
}

\section{Éric Gagnon}

Volume 44, numéro 1, 1995

Valeurs, pratiques, action sociale

URI : https://id.erudit.org/iderudit/706680ar

DOI : https://doi.org/10.7202/706680ar

Aller au sommaire du numéro

Éditeur(s)

École de service social de l'Université Laval

ISSN

1708-1734 (numérique)

Découvrir la revue

Citer cet article

Gagnon, É. (1995). Engagement social, engagement identitaire - Parcours de femmes. Service social, 44(1), 49-67. https://doi.org/10.7202/706680ar
Résumé de l'article

De la participation aux associations et groupes communautaires, on a souvent espéré une solution, sinon un correctif au bris des liens sociaux, en plus d'en faire un mode privilégié de participation politique. Ces questions sont ici reprises en tentant de comprendre les changements dans les modes de participation suivant l'itinéraire des personnes, leur âge et leur génération, en fonction des transformations culturelles venant modifier la mission des associations. L'analyse repose sur l'exemple d'une génération de femmes, qui ont aujourd'hui autour de soixante ans, travailleuses au foyer la plus grande partie de leur vie, et dont nous retraçons les trajectoires d'engagement, de la paroisse aux groupes politiques. 


\section{Engagement social, engagement identitaire Parcours de femmes}

Éric GAGNON

Chercheur postdoctoral Centre de recherche sur les services communautaires

Université Laval

Depuis son émergence au siècle dernier, la «question sociale» demeure étroitement liée à la vie associative, au dynamisme des groupements volontaires. De la participation aux associations, on espère une solution, sinon un correctif au bris des liens sociaux, à la disparition des normes communes garantes des solidarités, en plus d'en faire un mode privilégié de représentation politique et de formation d'un espace public. Ces attentes à l'endroit des associations ne furent certes pas constantes - l'État, pour un temps, fut préféré - et les types de groupements désirés ont varié : corporation professionnelle, syndicats, groupes d'intérêts ou d'entraide et ce que l'on rassemble aujourd'hui sous la dénomination "groupes communautaires». Ces variations sont d'ailleurs le signe de changements des rapports sociaux, des enjeux politiques et, par-delà, des représentations de la participation et du «social» lui-même. 
Permanence et transformations d'une interrogation et d'un projet, que manifeste le foisonnement récent d'études sur les groupes et associations de tout ordre, sur le bénévolat et l'engagement social, le don et I'entraide. On redécouvre ainsi des associations anciennes, "traditionnelles», tels les Cercles de fermières, leur trouvant des vertus jusque-là ignorées, et leur reconnaissant un rôle de précurseurs de la révolution tranquille et de la modernité. Même les groupes de loisirs apparaissent des lieux importants de solidarité et de prise en charge de la communauté par elle-même. La crise prolongée de l'Étatprovidence, une certaine désaffection du politique, le repli sur soi et le morcellement des identités motivent ces recherches sur les groupes comme médiateurs (possibles) entre les individus et la société, et, sur le plan historique, entre la tradition et la modernité, entre les solidarités anciennes et nouvelles. Mais tant d'attention à la participation dénote avant tout que celle-ci ne va pas de soi, qu'au-delà des solidarités de base, de l'entraide entre proches, la jonction de l'individu avec une communauté plus large demeure incertaine et que, par-delà la satisfaction des intérêts particuliers de leurs membres, les associations ne conduisent pas nécessairement à des solidarités plus vastes, à un sentiment de responsabilité sociale et à une interrogation politique sur le vivre ensemble.

$C^{\prime}$ est dans ce contexte qu'une étude sur l'engagement social des femmes de plus de cinquante ans nous a été commandée par l'Association féminine d'éducation et d'action sociale (AFEAS)' ${ }^{1}$. Devant le décrochage de femmes autrefois très actives dans le mouvement, des responsables de l'Association s'interrogeaient sur le devenir de l'engagement et sur les causes d'une certaine démobilisation, dont l'importance demeure d'ailleurs difficile à apprécier. Les femmes âgées veulent-elles et peuvent-elles encore jouer un rôle dans la société ? La retraite correspond-elle à un "engagement" dans les loisirs? L'AFEAS a voulu comprendre ce qui mène les personnes à renoncer à leur engagement, ainsi que les nouvelles formes que celuici peut prendre. Cette interrogation est liée à un souci d'assurer le dynamisme de l'organisation et le maintien du membership, mais elle est aussi née d'une préoccupation et d'une prise de position : s'engager $n^{\prime}$ est pas simplement un moyen de satisfaire ses propres besoins, mais aussi de s'ouvrir aux autres et d'envisager la société de manière globale; l'engagement social va donc au-delà de l'aide à la famille et aux proches, même s'il peut s'agir là d'une contribution sociale. $C^{\prime}$ est également plus que $d^{\prime}$ offrir des services à des clientèles spécifiques, plus que de défendre les intérêts de groupes particuliers. Le faire bénévolement est d'ailleurs le signe du désintéressement de la 
personne, l'indication qu'elle ne s'engage pas uniquement pour ses propres intérêts, et qu'elle est tournée vers la collectivité. L'engagement véritable ne saurait être une simple activité sociale, toute profitable qu'elle puisse être au bien-être des individus, mais une action sociale. Aussi, pour l'AFEAS, il est important d'en assurer la vitalité. Mais l'engagement ainsi conçu s'accorde-t-il encore avec les aspirations des femmes et correspond-il bien à la signification qu'elles donnent de leur participation dans une association?

Sur la base des résultats de cette étude, je veux ici reprendre ces interrogations, tenter de comprendre les changements dans les modes d'engagement, suivant l'âge des personnes, la période de leur vie et leur trajectoire, et en fonction des transformations culturelles affectant le rôle social des associations. Je le ferai à partir de l'exemple d'un groupe particulier, d'une génération de femmes, travailleuses au foyer ou dans une entreprise familiale la plus grande partie de leur vie, engagées dans leur paroisse plutôt que dans des associations professionnelles. Des femmes qui ont aujourd'hui autour de soixante ans et qui ont bien voulu retracer leur itinéraire d'engagement social $^{2}$. On verra que les organismes, ainsi que le type d'actions, sont variés et que la notion d'engagement peut prendre une signification très large ${ }^{3}$. Ces trajectoires peuvent nous faire avancer sur la question de la participation aux associations ou à des organismes comme les caisses populaires, les coopératives ou la paroisse, comme mode privilégié de solidarité et d'action sociale. Elles permettent l'observation de changements dans la participation et le rapport au politique liés à l'âge, à la génération et aux changements culturels, trois phénomènes que j'examinerai successivement. Nous verrons quelle ouverture aux solidarités et aux changements permet cette expérience identitaire qu'est l'engagement social.

Du comité de parents de l'école à la campagne de souscription pour un organisme de charité, de la bibliothèque municipale au comité de pastorale, de la maison d'hébergement pour femmes victimes de violence au comité d'accueil des nouveaux paroissiens, les femmes rencontrées ont œuvré de manière bénévole dans de multiples milieux. Mais des activités à caractère plus ludique, telle la chorale, à celles se rapprochant plus d'un travail, telle la participation au conseil d'administration de leur caisse populaire, les mêmes motivations sont toujours invoquées: d'abord le besoin de sortir de chez soi, de rencontrer des gens, de nouer des liens, d'accomplir une tâche valorisante surtout, d'obtenir une reconnaissance pour ce que I'on fait, de rendre ce que I'on a reçu (don et contre-don) en aidant 
ceux dans le besoin et de conserver un horaire souple et une autonomie dans la tâche.

Ces motivations nous les retrouvons dans toutes les études sur le bénévolat et sur la participation politique, comme "facteurs " prédisposant à l'engagement, mais leur seule énumération ne suffit pas pour comprendre ce que les choix signifient pour les individus qui les font. Ça n'explique pas non plus pourquoi certains se retirent, ni non plus pourquoi d'autres ne s'engagent jamais. Ces difficultés, nous pouvons les compenser en recomposant les trajectoires d'engagement des femmes, incluant les actions auxquelles elles ont renoncé, les organismes qu'elles ont quittés, en somme les choix posés à différents moments de leur vie. D'où l'intérêt d'interroger des femmes autrefois engagées, aujourd'hui retirées. Engagement et désengagement sont à comprendre ensemble, et c'est en retraçant des parcours qu'on comprend mieux ces choix.

Des mères de famille, travailleuses au foyer, dont la première motivation à s'engager fut le besoin de sortir de la maison, de rencontrer d'autres femmes, ont été ainsi rencontrées. L'arrivée des enfants, les travaux domestiques nombreux ne leur permirent pas une vie sociale très active au cours des premières années de leur mariage. La maison, le couple, les enfants accaparent le temps et les énergies, concentrent les préoccupations. Avoir soin d'une maison est une tâche ingrate qui n'a jamais de fin, et le besoin d'en changer se fait parfois pressant. Mais les enfants vont vieillir et commencer à aller à l'école: du temps se libère. C'est alors que des femmes commencent à participer à leur premier comité ou association.

Les comités d'école répondent particulièrement bien au besoin de voir du monde et à la gratification recherchée, tout en contribuant à l'éducation des enfants. Ces femmes n'ont alors pas le sentiment que l'engagement se fait au détriment du rôle parental; au contraire, il le complète. Participer au comité d'école, c'est s'assurer que les enfants reçoivent une éducation de qualité. Comme mères de famille et comme femmes qui auraient aimé étudier, l'éducation revêt souvent une très grande importance. Les enfants procurent ainsi une double motivation: on s'engage pour ne plus seulement s'occuper $d^{\prime}$ eux et en même temps pour mieux s'en occuper. La bibliothèque et les loisirs scolaires ont également procuré à des femmes une première occasion de s'engager, de se faire remarquer, de rencontrer des gens, de se faire offrir parfois une autre activité, d'autres responsabilités: comité de pastorale, campagne de souscription pour un organisme de charité, etc. 
D'autres vont débuter dans une association comme l'AFEAS, les Fermières ou les Filles d'Isabelle. Elles sont généralement invitées par une amie, une sœur ou une belle-sœur à se joindre au groupe. Elles $y$ vont pour rencontrer d'autres femmes ou pour se divertir. On y tisse de nombreux liens d'amitié. Une femme mariée, après avoir souligné l'importance de la cause des femmes défendue à l'AFEAS, parle de son groupe comme d'une "famille». Une autre parle de "l'engagement humain ", une expression qui associe le besoin de rencontrer des gens avec la responsabilité sociale. Les amies et amis, lorsqu'ils vivent des expériences difficiles telles que la maladie ou la mortalité, on les soutient souvent comme on le fait pour les membres de sa propre famille.

Ainsi les femmes viennent aux groupes et aux associations d'abord pour "elles-mêmes»; I'engagement vient après, à mesure que les enfants grandissent et qu'ils quittent la maison, à mesure qu'elles-mêmes prennent de l'assurance, que l'intérêt se développe. Les enfants devenus grands, en effet, elles quittent le milieu scolaire et les loisirs familiaux où elles ne se sentent plus directement concernées. De là, certaines passent dans d'autres organismes, se retrouvent dans des organismes communautaires ou à des tables de concertation régionale, parfois siègent à des conseils d'administration d'établissements publics. C'est la seconde étape en quelque sorte. Si elles le veulent, elles peuvent monter et faire une "carrière " de bénévole. "On vous appelle pour le comité d'école, puis on vous demande d'être secrétaire.... J'ai été là 11 ans!» L'AFEAS conduit à d'autres activités, d'autres engagements, du bénévolat; on se retrouve dans un réseau, sur une liste de personnes bénévoles potentielles et on se fait appeler pour diverses actions et divers organismes. Une travailleuse au foyer n'hésite pas à dire: "Nous aussi on a la double tâche avec tout le bénévolat qu'on fait. »

Le comité de surveillance de la Caisse populaire a souvent été la porte d'entrée des femmes au conseil d'administration de la Caisse: elles ont pris de l'expérience, acquis une formation, se sont fait remarquer et sont prêtes à accéder à un milieu jusque-là réservé aux hommes. D'autres se retrouveront au comité d'usagers de leur CLSC, quelques-unes feront de la politique municipale, comme conseillères municipales notamment. Le bénévolat, depuis le début du siècle, est demeuré un moyen pour les femmes d'élargir leur champ d'activité, hors de la sphère privée, au monde du travail (même s'il est non rémunéré $)^{4}$, conduisant par la suite plusieurs d'entre elles au féminisme, à l'action sociale ou à diverses formes d'engagement dans l'Église. 
L'engagement social procure une autonomie, mais il faut déjà avoir acquis une certaine autonomie pour s'engager, particulièrement vis-à-vis du conjoint. Et l'autonomie ne s'obtient pas toujours facilement. "Quand j'ai commencé à travailler, mon mari n'a pas aimé cela. Ça été un choc! J'ai brisé une image. " II faut lui faire comprendre le bien-être que procure l'engagement. Une femme a cette formule: "J'ai amené mon mari à choisir mon choix. " Pas seulement à le tolérer.

Plusieurs prétendent connaître des femmes qui n'ont pu faire partie d'associations à cause de leur conjoint, ou qui ont cessé d'en faire partie lorsque leur mari a pris sa retraite. Elles demeurent à la maison pour être disponibles, l'accompagner ou ne pas le laisser seul. Pour certaines, c'est un choix, comme on le verra plus loin. D'autres y seraient contraintes. Nous n'avons pas rencontré ces dernières, ne pouvant les rejoindre, puisqu'elles ne "sortent " pas. Il est difficile par conséquent d'apprécier cette réalité. Mais l'insistance avec laquelle on en parle est le signe sans doute de l'importance de la relation au conjoint pour toutes les femmes, engagées ou non. L'autonomie ici recherchée n'est d'ailleurs pas sans lien avec le besoin de reconnaissance et de valorisation ressenti par toutes.

Le rôle de mère, $\mathrm{s}^{\prime}$ accordent-elles toutes à dire, ce $\mathrm{n}^{\prime}$ est pas toujours gratifiant. Le travail au foyer $n^{\prime}$ est pas valorisé et il procure peu de reconnaissance. Ce que l'on fait en dehors de la vie familiale et du travail, dira l'une d'elles, "c'est un peu une récompense ». Une femme parle de la "chance» qu'elle a de faire partie du comité d'entraide de la paroisse. Aider les plus démunis, "ça me grandit». Elles accomplissent quelque chose qu'elles savent utile aux autres et même parfois indispensable à la société. "II n'y aurait pas de bibliothèque sans les parents bénévoles pour s'en occuper."

Les motivations égoïstes et altruistes se mêlent et se confondent en partie. On s'engage d'abord souvent pour la satisfaction de besoins personnels (sociabilité, estime de soi), mais ces besoins ne seraient pas satisfaits si l'action ne comportait pas une dimension altruiste: l'action est socialement valorisée et reconnue par les autres, parce qu'elle est désintéressée. Paradoxe: c'est justement en n'agissant pas uniquement dans son propre intérêt qu'on y trouve finalement son intérêt (la reconnaissance). "Le bénévolat, $c^{\prime}$ est un peu pour se réaliser ou réaliser ses idéaux." Notons que l'emploi des termes "égoïste» et "altruiste » ne revient pas à juger de la sincérité et des intentions véritables des personnes, dont nous ne sommes jamais certains. II s'agit de catégories culturelles qui me servent, et dont mes informatrices se servent ${ }^{5}$, pour distinguer deux types de motivations 
possibles pour les individus, auxquelles correspondent d'ailleurs deux orientations possibles pour un organisme (j'y reviendrai). Plutôt que des jugements sur les motivations "réelles" des personnes, ce sont des références, des idéaux qui servent à comprendre et à s'orienter.

L'engagement est contraignant comparativement aux loisirs : "Souvent il faut que tu te marches sur le cœur, que tu te forces. Si tu attends que ça te tente, tu ne le feras jamais. » En comparaison avec I'engagement familial, l'engagement social comporte cependant une part de liberté. La famille est un devoir, quelque chose qui «va de soi », de "naturel », donc d'obligatoire, même si ça procure souvent du plaisir (notamment les soins aux petits-enfants). Ce caractère désintéressé de l'engagement le distingue à la fois du loisir et de l'engagement familial: on s'y contraint... librement.

On donne quand on a le sentiment d'avoir autrefois reçu ou de recevoir aujourd'hui quelque chose en échange. La réciprocité est nécessaire. L'engagement est un don: on en attend un retour, ou on donne en retour de ce qu'on a reçu par le passé. Le contre-don s'obtient généralement par une forme de reconnaissance ou de satisfaction personnelle. La formation reçue peut aussi valoir dans l'échange. Ce que ces femmes ont appris leur a servi par la suite: " Tout ce que j'ai fait, ça m'a servi après. Ce que j'ai fait m'a toujours été rendu. »En trouvant de l'aide et de l'amitié dans les groupes et dans les organisations, on contracte alors une dette. Ainsi, une femme raconte qu'à une période difficile de sa vie, où elle était déprimée, elle a trouvé dans le Cercle des Fermières des amies qui l'ont aidée et "sauvée". Même si aujourd'hui l'atmosphère du cercle a changé, elle y demeure attachée. Sa fidélité au Cercle est un peu le paiement de sa dette. Même attitude chez une femme autrefois très active, aujourd'hui désengagée, mais qui continue de payer sa cotisation: "Je suis redevable à l'organisme.»

L'engagement, on l'a vu, est lié à l'âge et aux périodes de la vie. En passant à une nouvelle étape de l'itinéraire des femmes, on verra qu'il est également lié aux conditions particulières de chaque génération.

Si chez certaines femmes l'engagement bénévole dans les groupes et associations demeure tout au long de leur vie, pour la majorité, tôt ou tard, vient l'heure du désengagement, complet ou partiel. Un besoin de changement se traduit quelquefois par un retour aux études, un peu plus souvent par l'intégration au marché du travail. 
Les enfants élevés, les femmes se trouvent un emploi; elles deviennent en même temps moins disponibles pour les activités bénévoles. Pour certaines leur engagement a même favorisé l'obtention d'un emploi, par les contacts qu'il aura permis d'établir ou l'expérience qu'il aura procurée. Leur engagement aura été pour elles le moyen de développer cette carrière.

Mais il n'y pas que celles qui gagnent le marché du travail qui se désengagent. Le décrochage $n^{\prime}$ est pas qu'une question de disponibilité. Elles ont aussi moins le goût. Après l'engagement intense, on passe à autre chose et on ne veut souvent plus y revenir. On veut du temps à soi, du temps avec son conjoint, un repos mérité. La retraite du conjoint ou son décès constitue d'ailleurs souvent un moment important dans une trajectoire d'engagement: la vie et les activités font l'objet d'une réévaluation et d'une réorganisation ${ }^{6}$.

De nombreuses femmes nous ont parlé d'un besoin d'horaire flexible, variable, même si elles demeurent présentes dans l'organisation; un besoin qui vient avec l'âge, après les années très chargées consacrées à l'éducation des enfants, au travail parfois, et aux divers engagements. Accompagner des personnes âgées à l'hôpital est ainsi préféré à des postes de responsabilités dans une association. Les femmes ne veulent plus s'engager longtemps d'avance et à long terme, elles ne veulent plus prendre de responsabilités. On ne veut plus se sentir contrainte comme on l'était sur le marché du travail ou lorsqu'on élevait sa famille. Mais au-delà de la question du temps, il y a ce besoin de faire quelque chose pour soi. Le travail rémunéré est pour plusieurs une activité pour soi. Pour d'autres, se tourner vers soi, ce sera entreprendre une démarche spirituelle: s'intégrer à un groupe de prière, de croissance personnelle, ou consacrer son temps à la lecture, aux arts, à la discussion avec des amies. "Je n'ai plus d'agenda; j'ai un journal personnel! » Après I'engagement social, elles se tournent vers l'engagement spirituel, une démarche éminemment individuelle, même si d'autres les accompagnent. Pour d'autres encore, ce sera tout simplement les loisirs.

Les problèmes de santé viennent limiter les activités, surtout après 65 ans. Mais la santé est rarement seule en cause. Ainsi cette femme, membre de I'AFEAS pendant près de vingt ans, qui a fait du bénévolat au comité d'école et ailleurs, et qui a quitté l'Association lorsqu'elle a intégré le marché du travail. En plus des raisons de santé, elle dit n'avoir plus autant besoin de sortir, avoir moins besoin de formation avec toute l'information qu'on peut aujourd'hui recevoir à la maison, et avoir moins besoin de contacts puisqu'elle en trouve ailleurs. Elle peut sortir davantage avec son mari depuis qu'il a pris 
sa retraite et que les enfants sont partis. Autrefois, ils sortaient chacun à tour de rôle, l'un des deux devant garder les enfants. Et puis elle avoue avoir perdu un peu "la foi » dans l'engagement social; elle n'est plus aussi motivée qu'avant. II revient aux jeunes de reprendre le flambeau.

Tous ces besoins nouveaux et ces attitudes nouvelles, que signale parfois la retraite et que condense la formule "pour soi », signifient chez plusieurs femmes l'aboutissement de ce qu'elles appellent une "démarche». Le besoin de sortir, de créer des liens, de réaliser des activités valorisantes, de parfaire leur formation, qui les avait conduites à l'engagement, est en partie satisfait. L'engagement $n^{\prime}$ est plus aussi nécessaire, il ne se fait plus aussi pressant. L'objectif d'accroître la confiance et l'expression de soi, d'une association comme l'AFEAS, a été largement atteint, avec l'effet imprévu de conduire à un certain désengagement. D'ailleurs, si l'un de ces besoins se manifeste encore, et même si le contexte est différent, l'engagement peut demeurer la manière de le satisfaire. Des femmes poursuivent leur engagement, entre autres raisons, pour fuir la solitude causée par le décès de leur mari.

La fin de leur démarche d'expression et de valorisation de soi $s^{\prime}$ exprime également par une certaine fatigue et un éloignement de la politique. L'engagement peut prendre une grande place et devenir envahissant. II peut occuper tout le temps et tout l'esprit. Lutter pour une cause peut devenir mentalement épuisant: "C'est fatiguant de s'indigner toujours, de haïr. »Le conflit, le ressentiment sont destructeurs. Travailler pour la condition féminine, diront certaines, ce n'est pas toujours facile: on ne voit pas toujours les résultats. Les mentalités, c'est difficile à changer. Les femmes qui ont progressé dans une organisation en occupant des postes de responsabilité plus élevés, au niveau provincial par exemple, lorsqu'elles retournent à la base perçoivent un écart: elles trouvent les femmes moins conscientisées, moins autonomes, moins revendicatrices qu'elles-mêmes le sont devenues.

Si l'engagement se poursuit, on s'oriente encore davantage vers la paroisse que le politique, davantage vers l'assistance à des personnes dans le besoin que vers la formation et la conscientisation: comme marguillières, par exemple, ou au sein d'un comité d'accueil pour les nouveaux arrivants. Une femme, enseignante à temps partiel, demeure membre de I'AFEAS et du Cercle des Fermières par solidarité, mais s'implique plutôt aujourd'hui dans un organisme pour les jeunes de 18 à 30 ans qui se rencontrent pour discuter sur leurs relations avec leurs amis, leur parents. L'organisme veut servir d'intermédiaire, aider les jeunes et leurs parents à se parler. 
L'engagement à un niveau plus local et orienté vers les services à la communauté ou aux démunis n'est pas uniquement lié à l'âge. En fait, ce type d'engagement, les femmes l'ont pratiqué à tout âge. Seulement, passé la cinquantaine, il semble encore mieux convenir, mieux satisfaire les nouveaux besoins et les nouvelles exigences. Lorsqu'elles étaient plus jeunes, I'entraide a conduit les femmes à I'engagement; aujourd'hui c'est l'engagement qui les conduit à I'entraide. Le "bénévolat de service» serait donc souvent préféré au "bénévolat d'engagement", pour reprendre la distinction et les termes d'une des femmes rencontrées, pour qui seul la deuxième forme est un véritable engagement social, c'est-à-dire pouvant mener au changement. Pour la plupart des femmes, toutefois, ces services sont une forme d'engagement. Un changement dans la nature politique de l'engagement est cependant repérable. Nous y reviendrons.

Considéré dans l'ensemble de leur itinéraire, I'engagement social semble avoir été pour beaucoup de femmes un moyen de réconcilier les changements avec ce qu'elles croient devoir durer. L'AFEAS, par exemple, a joué ce rôle pour les femmes travailleuses au foyer principalement, pour des mères de famille, à la suite des transformations survenues dans la société québécoise depuis 30 ans: modifications des rôles sexuels, féminisme, entrée massive des femmes sur le marché du travail, augmentation des divorces, baisse de natalité, affaiblissement des pratiques religieuses, etc. La participation à l'AFEAS les a aidées à préserver et à changer en partie leur identité, à valoriser leurs activités, à acquérir des compétences, à comprendre les changements et à se situer par rapport à eux. Les associations sont l'une des réponses modernes aux problèmes posés par les transitions, lorsqu'il y a nécessité de reformuler des normes. L'engagement social est l'occasion de distinguer ce que l'on juge important de ce qu'il l'est moins, de conférer une valeur à des gestes et à des situations. Dans une situation où l'identité devient incertaine, où elle est ébranlée, remise en question, l'engagement permet de reconnaître ce que l'on veut préserver et ce que l'on veut changer. D'ailleurs, les changements que connaissent les rôles féminins, particulièrement depuis une trentaine d'années, $n^{\prime}$ ont pas conduit au remplacement $d^{\prime}$ un modèle unique par un seul nouveau modèle, mais bien plutôt par une variété de modèles, qui coexistent plus ou moins bien. II faut trouver une manière de concilier des valeurs et des conduites anciennes et nouvelles, auxquelles on tient ${ }^{7}$. Les groupes dans lesquels les femmes s'engagent peuvent y contribuer, en favorisant l'assimilation d'idées nouvelles ou en confirmant les individus dans leur choix. 
À I'AFEAS, plusieurs cherchaient à changer la condition des femmes sans dévaloriser ce qu'elles étaient; réconcilier le passé et le présent. Pour plusieurs membres, comme le montre l'enquête de 1980, il s'agissait d'améliorer leur rôle traditionnel plutôt que de changer de rôle. Les femmes ont de cette manière participé aux changements, tout en cherchant à défendre des valeurs, à défendre ce qu'elles étaient ou avaient été. Défendre la famille tout en libérant les femmes. Une manière d'apprivoiser les changements et $d^{\prime} y$ participer. Les Dames de Sainte-Anne ou le Mouvement des femmes chrétiennes furent également un lieu d'échanges, de discussions et de formation, où l'on se préoccupait principalement du mieux-être de la famille et du sort de la communauté : une autre manière d'apprivoiser le changement, en assurant une continuité. Ces organismes jouent le rôle de corps intermédiaires, non seulement sur le plan politique (groupe de pression, de représentation), mais aussi sur le plan chronologique, pourrions-nous dire, pour la mémoire et pour la continuité; médiation également dans la formation des identités individuelles et collectives $^{8}$.

Les itinéraires de ces femmes, trop rapidement ici retracés, conduisent à relativiser l'opposition entre les motivations égoïstes et altruistes et à relativiser également l'opposition posée au départ entre activité sociale et action sociale. Vues comme un mode privilégié de construction et d'affirmation identitaire par lequel les solidarités prennent finalement leur sens - la personne s'insère dans un groupe et, sur le plan symbolique, dans la société tout entière - les visées collectives de changement et les visées personnelles de bien-être et d'affirmation apparaissent solidaires. Et, de fait, on passe souvent imperceptiblement de I'action à l'activité sociale, et inversement: de la fête paroissiale aux pressions exercées sur le député, à la campagne de la Croix-Rouge et aux discussions avec des amies sur la condition féminine.

Les multiples facettes de l'engagement mettent en cause l'identité de la personne: la cause défendue repose sur des valeurs qui lui sont chères. Comme lieu d'expression et de reconnaissance de soi, I'association renforce la confiance et l'estime de soi; le sentiment d'utilité améliore également l'image de soi; le bénévolat est l'occasion de créer des liens, mais aussi de "signifier " ces liens, d'exprimer son appartenance à la communauté; il permet l'adoption de nouveaux rôles et $d^{\prime} u n$ nouveau statut à diverses étapes de la vie (arrivée et départ des enfants, retraite). La question des valeurs ne se résume donc pas à celles qui fondent l'action du groupe (charité, justice), mais elle s'étend à toutes celles qui structurent la personna- 
lité des membres et commandent ses relations aux autres. Les motivations à l'engagement dépassent le sentiment d'utilité et de bien-être psychologique sur lequel on accorde tant d'importance dans les écrits sur le bénévolat. En fait, l'examen d'itinéraires montre l'insuffisance des efforts pour identifier les "facteurs» favorisant la participation, afin $d^{\prime}$ 'en mesurer $I^{\prime}$ " influence» respective ${ }^{9}$. Relever de manière séparée et hors contexte les "conditions propices " à l'engagement n'accroît pas beaucoup notre compréhension du phénomène. II faut replacer les choix dans une trajectoire, un milieu social et des changements l'affectant. D'ailleurs, les générations de femmes suivant celle que nous avons étudiée ne pourront suivre des parcours identiques, ni être motivées par les mêmes "facteurs". Si les besoins de reconnaissance et de sociabilité vont demeurer, ils s'affirmeront - s'affirment dès à présent - sans doute autrement. Le travail à l'extérieur du foyer et la double tâche excluent à eux seuls de semblables trajectoires.

À propos de femmes, d'abord utilisatrices d'un service fourni par un groupe de femmes, puis bénévoles au sein de cet organisme, G. Côté et M.-A. Couillard (1995: 117) font cette remarque: "Elles disent [...] s'engager afin de "repayer" à la collectivité ce qui a été "reçu". Cette formulation laisse croire qu'il s'agit de rembourser une dette, comme si l'on pouvait estimer ce qui fait l'objet du don, alors qu'en réalité il ne fait aucun doute que, ce dont il est question, c'est une prise de conscience qui modifie le soi et le rapport au milieu dans lequel il évolue.» Et les auteures de préciser: "Parce que cette prise de conscience a été rendue possible par l'engagement $d^{\prime}$ autres femmes, il leur semble juste de s'engager à leur tour dans un processus qui va au-delà de l'action bénévole. » Cette interprétation a le mérite de faire ressortir la transformation qui s'opère dans l'engagement: les motivations qui y conduisent changent en cours de route du fait d'une transformation de l'identité de la personne. Leur engagement $n^{\prime}$ est donc pas un simple remboursement, qui équivaut à l'aide reçue. Les expressions "en réalité " et "il ne fait aucun doute "soulèvent cependant une interrogation. Outre leur assurance quant à la supériorité de leur interprétation, les auteures affichent une confiance quant au caractère social, voire politique de l'engagement de ces femmes (I'" au-delà de l'action bénévole»). Le travail sur soi, I'entraide, et même le sentiment de travailler pour une cause juste et qui les dépasse, ne laissent pas préjuger de la portée sociale et politique de leur participation et de l'action du groupe dans son ensemble. C'est la question qu'il me reste à traiter.

L'engagement est lié à l'âge et aux conditions propres à chaque génération; il est également associé à des transformations culturelles et politiques qui pourraient en modifier la nature et la portée. Si une 
orientation vers l'actualisation de soi et une orientation vers le service à la société ne sont pas en concurrence, du moins à certaines étapes de la vie, comme on l'a vu, le mode de prise en charge des problèmes et de formulation des enjeux pourrait bien être en train de changer.

Décrivant la phase de désengagement, je notais un éloignement du politique et une préférence pour les services communautaires. L'engagement ne conduit pas nécessairement à la politique, même si la politique, surtout municipale, attire quelques femmes. Le moins qu'on puisse dire, c'est que la plupart des femmes rencontrées sont tièdes vis-à-vis de la politique. Chez elles nous retrouvons, dans leur attitude à l'égard de la politique, plusieurs éléments que nous venons $\mathrm{d}^{\prime}$ aborder, mais en contrepoint. En effet, ce qui déplaît en politique, c'est la compétition, les motivations trop intéressées: on travaille pour des votes, pour des emplois, pas pour les autres. La politique, $c^{\prime}$ est la rivalité, la bataille, les conflits, les manipulations, la défense des intérêts personnels. "C'est un peu une jungle. " Un engrenage conduisant à la corruption ou au mensonge dans lequel se font prendre même les meilleurs. "J'ai plus ou moins confiance: ce ne sont pas les plus compétents qui vont en politique, ce sont ceux qui crient le plus fort.»

Une femme dont le mari a été député garde un mauvais souvenir de la politique, des tactiques et des stratégies, des attaques touchant la vie privée dont ils furent l'objet: "C'est ingrat. » Nous retrouvons ici encore la question de la reconnaissance. Elle préfère I'AFEAS: "C'est de la politique qu'on peut aimer faire. " C'est faire de la politique "en douceur ». "Notre politique est familiale», dira une dame, affirmant par là le caractère désintéressé et communautaire, autant des causes défendues que du mode d'action privilégié. Comparant son expérience en politique municipale avec sa participation au conseil d'administration d'une caisse populaire, une femme soutiendra qu'en politique c'était chacun pour soi, tandis qu'à la Caisse ils formaient une équipe. De cette seconde expérience, elle conserve d'ailleurs un excellent souvenir. Si plusieurs ont fait du travail électoral, c'était d'abord pour rendre service à une amie qui sollicitait leur collaboration. Certains témoignages sont éloquents: "J'ai travaillé pour une élection. Je distribuais des tracts les samedis. On organisait des réunions. Une semaine après l'élection tu rencontres le député au magasin et il ne te reconnaît même plus! Est-ce parce que j'étais bénévole? Si j'avais été payée peut-être qu'il m’aurait 
reconnue..." Nous retrouvons ici le besoin de reconnaissance... dans sa manifestation la plus élémentaire!

Il a été peu question dans nos entretiens d'engagement dans des organismes de défense des droits, tels ceux des locataires ou des minorités. Par contre, il a souvent été question d'engagement dans la paroisse: communion à domicile pour des personnes malades, comité d'accueil, comité de pastorale ou de liturgie, responsabilités de marguillières ou de servantes de messe. L'une raconte avoir participé à la mise sur pied d'une petite bibliothèque dans la paroisse: on ramassait des livres; les gens donnaient 25 cents par emprunt et avec cet argent on achetait d'autres livres. Pendant un an, en attendant le remplacement du curé, un groupe de femmes de Chicoutimi s'est occupé de la paroisse. La participation à la vie communautaire leur apparaissait - et leur apparaît encore - comme une forme d'engagement social, même si ce n'était pas directement orienté vers le changement social. Cela n'excluait pas un engagement à l'égard de la condition féminine, mais un engagement plus spécifique, plus "localisé » peut-être: les femmes pauvres, les femmes dans l'Église, par exemple.

En somme, ce que l'on reproche à la politique, c'est d'être dépourvue des attraits de l'engagement que nous avons précédemment relevés: amitié, reconnaissance, don, aide à ceux qui en ont véritablement besoin. En outre, l'action politique ne donne pas de résultats immédiats, ni toujours tangibles, tandis que l'action locale et communautaire procure une aide concrète et immédiate à des individus bien identifiés. La politique apparaît souvent comme provoquant la division et menaçant les valeurs. Elle semble également exiger un savoir. L'action communautaire, remarque Godbout (1987), donne plutôt un sentiment de solidarité, de cohésion sociale, et met à profit l'expérience des gens. Pour ces raisons, l'action politique n'apparaît pas toujours la plus intéressante ni la plus efficace.

Mais ne peut-on pas aller plus loin et se demander si cette méfiance à l'endroit de la politique et l'intérêt pour le communautaire ne seraient pas le symptôme d'une mutation de la culture et du politique. Comme I'ont ailleurs relevé M.-A. Couillard et G. Côté (1994), il existe une tendance vers les "alliances ponctuelles » sur des enjeux précis, pour l'atteinte d'objectifs particuliers, moins englobants. Un déplacement au cours des années dans le domaine d'action investi par les groupes de femmes a été remarqué par F.R. Ouellette (1990): $d^{\prime}$ abord le champ de la socialisation (redéfinitions des rôles sociaux), puis le champ politique (revendications et représentations à l'échelle provinciale) et maintenant le champ des services (aide aux victimes 
et aux démunies sur le plan local ou régional). N'avons-nous pas entendu souvent mentionner les maisons d'hébergement pour femmes victimes de violence comme lieu nouveau d'engagement? À cela, il y a plusieurs raisons, à commencer par les critères de reconnaissance et de financement du gouvernement, qui incitent les groupes à " cibler » leur clientèle et à aller du côté des services comme partenaires des services publics.

Il y a sûrement plus encore, peut-être le passage d'un mode privilégié $d^{\prime}$ association à un autre. La tendance, selon Jacques lon, serait d'aller d'une forme associative contractuelle, volontaire et militante, vers une forme "sociabilitaire», subjective et limitée, les syndicats et les grandes fédérations associatives représentant la première forme, les groupes d'entraide et les services communautaires, la seconde. Dans I'association de type contractuel, les individus sont réunis pour la défense des mêmes intérêts collectifs, chacun mettant de côté ses intérêts particuliers et attaches spécifiques. La participation suppose un "assentiment global à un système de valeur » (Ion, 1990: 177) ; I'implication identitaire est maximale, mettant en cause la vie de la personne en son entier - définie comme citoyen, travailleur ou femme - et sa place dans la société. Autrement dit, la cause défendue est coextensive à l'ensemble de la société (Fortin). Dans sa forme sociabilitaire, par contre, l'engagement dans une association est plus restreint, même s'il peut être vécu intensément. $N^{\prime}$ est alors engagé qu'un aspect de l'identité des participants: le fait d'être handicapé, chef de famille monoparentale ou résident d'une zone où l'on veut entreposer du BPC, par exemple. L'individu n'est pas défini comme citoyen, mais comme usager. La participation renvoie cette fois-ci aux liens concrets et particuliers de la personne ${ }^{10}$. Au premier type d'association correspond une phase de "subjectivation " dans les transformations du rapport entre l'individu et la société: I'affirmation de chaque individu comme sujet de droit, libre et égal aux autres; entité abstraite et délocalisée. Le second correspond à une phase $\mathrm{d}^{\prime}$ " individualisation», d'affirmation des différences et particularités de chacun ${ }^{11}$.

Michel Freitag qualifie, pour sa part, les deux phases de "moderne " et "postmoderne ». L'égalité des droits et l'égalité devant la loi dans la modernité font de l'individu un sujet politique, et de la communauté "une communauté de mobilisation politique». Les associations assurent une médiation et la participation aux délibérations collectives, par l'entremise de représentants. Cette forme d'identité politique est cependant menacée de dissolution, devant la multiplication des identités partielles, parfois transitoires, fondées sur 
des affinités, le partage d'une expérience existentielle ou d'un mode de vie. Les modes de participation politique s'en trouvent modifiés: au gré des rapports de force et des jeux d'influence, les groupes cherchent à faire reconnaître la spécificité de leurs membres, travaillent à la défense d'intérêts particuliers et à l'obtention de services, de ressources ou de mesures législatives pour une catégorie d'usagers. De ce changement, l'État-providence est en partie responsable, fractionnant les revendications politiques en une série de groupes $d^{\prime}$ intérêts et de clientèles spécifiques, dont il cherche à satisfaire les demandes. Cette "gestion du social » conduit à l'identification et à la défense d'intérêts toujours plus sectoriels, particuliers et locaux. Mis en concurrence, ils doivent se faire entendre, convaincre de la légitimité de leurs revendications, de l'urgence de l'appuyer.

G. Côté et M.-A. Couillard (1995) ont souligné une difficulté que pose la rémunération des permanents dans les groupes de femmes, et les embarras qu'elle crée. Est-ce encore de l'engagement social lorsqu'on est payé pour le faire? La gratuité de l'action demeure encore pour plusieurs le signe d'un désintérêt et d'une orientation vers les autres, comme je le notais en début d'article. Indice d'une professionnalisation, la rémunération des permanents (qui furent souvent d'abord bénévoles) pourrait être aussi l'indication d'une orientation vers les services spécialisés, ce qui expliquerait aussi l'embarras qu'elle suscite.

Par certains côtés, on semble toujours participer à l'avènement et à la généralisation du sujet de droit, les revendications faisant accéder à ce statut des catégories d'individus tenues jusqu'ici à l'écart. Mais tendanciellement, $c^{\prime}$ est à l'affirmation des différences et d'une identité particulière que les groupes s'emploient; surtout, ils ne produisent plus une intelligibilité nouvelle de la société, ils n'engagent plus une réflexion sur les finalités et les modalités de la vie en commun, comme on a pu et on continue de le faire à I'AFEAS. Or, il $n^{\prime} y$ a pas de sujet politique véritable sans un travail d'interprétation de la société globale. S'engager dans une cause avec beaucoup $d^{\prime}$ intensité, car elle touche une dimension existentielle forte, $n^{\prime}$ est pas nécessairement s'engager tout entier, ni engager tout l'entier, $\mathrm{c}^{\prime}$ està-dire la société dans son ensemble et les finalités communes. Si le rejet de la politique $n^{\prime}$ en est pas un du politique, il pourrait bien y être involontairement associé.

On comprend que, dans la première forme associative, la question de la représentativité et du nombre d'adhérents soit fondamentale, mais qu'elle soit secondaire dans la deuxième forme. Et c'est bien le souci de l'AFEAS, forme politique ou moderne d'association, d'accroître son membership, de demeurer un représentant reconnu 
des femmes; d'où son inquiétude quant au devenir de l'engagement social, et la recherche qu'elle nous a commandée...

\section{CONCLUSION}

Le rôle des associations, de l'avis de plusieurs, aurait changé au cours des années. D'instrument au service d'un projet pédagogique et social global (éduquer, conscientiser, former en vue de transformer la société), elles seraient davantage devenues un moyen d'expression et d'accomplissement de soi pour des individus appartenant à des groupes particuliers. Les associations contribueraient autant à la socialisation qu'à l'individualisation de leurs membres, chacun d'entre eux $s^{\prime}$ en servant pour se forger une identité propre. Nous avons amplement reconnu cette nouvelle fonction dans les témoignages entendus. Mais dans leur itinéraire les femmes rencontrées semblent souvent avoir suivi dans un premier temps le chemin inverse : $d^{\prime}$ abord moyen $d^{\prime}$ expression et d'affirmation de soi, leur participation les a conduites à l'action sociale.

Mais l'imbrication des motivations égoïstes et altruistes, de l'activité et de I'action sociales, qui nous ont paru dans un premier temps la chance d'une politique, et ce rôle médiateur des associations à l'égard des changements, de l'effritement des solidarités et de la rupture identitaire pourraient bien voir leurs effets se renverser: trop centrée sur soi et sur ses particularités, la participation aux associations menace de conduire à une dissolution du politique. II a souvent été fait mention que, dans les associations, sociabilité, identité et solidarité vont de pair: on aide et on fréquente ses "semblables" (famille, voisins, gens de même milieu). N'est-ce pas ce qui freine justement la solidarité, la limitant à un cercle étroit, en même temps que ce qui la rend possible, en favorisant la vie associative?

Dans notre culture, où l'autonomie prend tant d'importance, à la fois condition, moyen et visée des aspirations les plus grandes, nous avons tendance à opposer (tout au moins à distinguer) vie privée et vie publique, individualité et socialité, affirmation de soi et solidarité, autonomie et intégration au groupe. Mais nous avons vu que dans une recherche identitaire à travers l'engagement social, ces oppositions s'estompent ou s'atténuent: I'action pour autrui et I'action pour soi sont solidaires. Cette convergence varie cependant selon les périodes de la vie, les générations certainement, les tendances culturelles sans doute. Mais des tendances ne sont pas des fatalités. 


\section{Notes}

1. L'AFEAS est formée d'un réseau de 23000 femmes réunies dans 500 groupes locaux à travers la province. La discussion et l'information diffusée au sein des groupes sur différents sujets touchant les femmes ont pour but l'éducation des membres, la réflexion individuelle et collective ainsi que des actions dans le milieu, afin d'améliorer la condition des femmes. Sur les plans régional et provincial, des actions sont entreprises pour mieux comprendre une situation ou un problème et faire pression sur les autorités concernées en vue d'obtenir un changement.

2. Cette recherche a été conçue et planifiée en étroite collaboration avec un comité de I'AFEAS composé de $\mathrm{M}^{\text {mes }}$ Marie-Paule Godin, Yolande Dubé, Marielle Dumont, Yolande Haines, Michelle Houle-Ouellet et Lise Tremblay-Cournoyer. Sept entrevues de groupe ont été réalisées à Cacouna, Chicoutimi, Montréal, Québec, Trois-Rivières et Victoriaville, en janvier, février et mars 1995 avec une cinquantaine de femmes, la plupart engagées à divers titres par le passé, dont une majorité aujourd'hui retirées en partie ou en totalité de leurs actions bénévoles. J'ai rencontré des femmes de milieux sociaux assez diversifiés. Trois groupes réunissaient des femmes habitant une grande ville ou sa banlieue (Montréal, Trois-Rivières, Québec), deux groupes réunissaient des femmes de milieu rural (des régions de Rivière-du-Loup et de Victoriaville) et un groupe était formé de femmes d'une ville moyenne en région (Chicoutimi). Une dernière rencontre regroupait des femmes des quatre coins de la province, autrefois très engagées dans I'AFEAS, et aujourd'hui retirées.

3. Ainsi la chorale paroissiale, comme service à la communauté et par sa dimension religieuse (partage et enseignement de valeurs), peut-elle être vue, par les femmes ellesmêmes ou par l'observateur, comme un engagement social.

4. Pour un bel exemple, voir le livre d'Aline Charles sur le bénévolat hospitalier.

5. Du moins usent-elles d'expressions équivalentes, telles « je le fais pour moi » et « je le fais pour les autres".

6. Notons que, pour d'autres femmes, la retraite ou le veuvage marquera plutôt le début de leur engagement.

7. Sur ce point, voir l'étude de Colette Carisse et Joffre Dumazedier.

8. Ce ne fut cependant pas toujours facile, ni recherché par toutes les femmes. "J'ai quitté rapidement l'AFEAS, dira l'une d'elles. Je ne voulais plus entendre parler de la famille. Je venais de finir d'élever mes enfants et je voulais passer à autre chose. Ce n'étaient plus mes affaires. »

9. On établit des fréquences, puis des corrélations entre les conduites et un certain nombre de variables sociodémographiques (sexe, âge, nombre d'enfants, occupation...), sans parvenir toujours à les expliquer et surtout sans établir de liens entre les différents facteurs et motivations. Par ailleurs, si l'on connaît assez bien les motivations de ceux et celles qui s'engagent grâce aux études sur le bénévolat, en revanche on connaît très mal ce qui retient les autres de le faire. On s'emploie à faire la démonstration que la participation est une bonne chose pour les individus et les personnes âgées en particulier (estime de soi, identité), sans parvenir à expliquer pourquoi la plupart de ces personnes préfèrent cependant les groupes de loisir, moins exigeants. Estce parce qu'elles ne savent pas ce qu'elles manquent, qu'elles n'en sont pas conscientes? Ou est-ce parce qu'elles cherchent autre chose ? II y a comme un hiatus: elles ont toutes les raisons de s'engager, mais seule une minorité d'entre elles le fait.

10. Il va sans dire que ce sont des idéaux-types, jamais pleinement réalisés, parfois en concurrence, et souvent se chevauchant au sein d'un même groupe.

11. Pour la France, Ion (1981) diagnostique dans le champ socioculturel un passage très rapide, en une trentaine d'années, "d'une problématique du citoyen à une problématique de la personnalité » (p. 37). 


\section{Références bibliographiques}

CARISSE, C. et J. DumAZEDIER (1975). Les femmes innovatrices. Paris: Seuil. CHARLES, A. (1990). Travail d'ombre et de lumière. Le bénévolat féminin à I'Hôpital Sainte-Justine, 1907-1960. Québec: IQRC.

CÔTÉ, G. et M.-A. COUILLARD (1995). "Itinéraires individuels pour un projet collectif: s'engager dans un groupe de femmes de la région de Québec », Recherches féministes, vol. 8, $\mathrm{n}^{\circ} 2$ : 107-125.

COUILlard, M.-A. et G. CÔtÉ (1994). "Solidarités de genre et pouvoir de femme », dans F.-R. Ouellette et C. Bariteau (dir.), Entre tradition et universalisme, Québec: IQRC, p. 379-396.

ION, J. (1981). "De la formation du citoyen à l'injonction à être soi », Espaces et sociétés, $\mathrm{n}^{\circ}$ 38-39 : 37-45.

ION, J. (1990). "Les trois formes de la sociabilité associative", dans R. Levasseur (dir.), De la sociabilité. Montréal : Boréal, p. 169-182.

FORTIN, A. (1991). "La participation: des comités de citoyens au mouvement communautaire », dans J.-T. Godbout (dir.), La participation politique. Québec: IQRC, p. 218-250.

FreitaG, M. (1992). "L'identité, l'altérité et le politique », Société, nº 9 : 1-55.

GAGNON, E. (1996). De l'activité sociale à l'engagement social. Montréal: Association féminine d'éducation et d'action sociale.

GodBOUT, J.T. (1987) «La communauté retrouvée? », Recherches sociographiques, vol. XXVIII, n²-3: 407-414.

OUELLETTE, F.-R. (1990) «Les regroupements de femmes dans les années 1980 », dans M.-M. T. Brault et L. Saint-Jean (dir.), Entraide et associations. Québec: IQRC, p. 73-94. 